\title{
Possible roles of remotely operated underwater vehicles (ROV) and robotics in mariculture of the future
}

\author{
JENS G. BALCHEN \\ Keywords: Mariculture, Aquaculture, Robotics, ROV, Control, Fish behaviour. \\ The paper surveys some possible future trends in mariculture technology emphasiz- \\ ing new principles for controlling animal motion. Against this background possible \\ applications of remotely operated underwater vehicles and robotics are reviewed.
}

\section{Introduction}

The engineering aspects of traditional mariculture (aquaculture) have been confined to such disciplines as civil engineering, for the design of mechanical enclosure systems like floating cages and ponds, and hydraulic engineering for the design of pumping and water treatment systems. (Osborne et al. 1990) It appears that the main tasks of the engineers have been to optimize certain given engineering concepts rather than search for new concepts with possibly better overall performance. An example of this is the application of a floating cage enclosure system for fish farming, a system with a rather limited volume $\left(8000-12000 \mathrm{~m}^{3}\right)$ made of standard net material. This technology has some apparent advantages but also some very serious disadvantages. An obvious alternative is to abandon the mechanical enclosure system and concentrate on other ways of controlling the motion of fish. One way which has been suggested and partly investigated in Norway is to utilize natural coastal formations such as bays, fjords and sounds together with different kinds of nonmechanical barriers to keep the fish in captivity, but providing a much larger volume. The nonmechanical barriers which have been tested are electrical, high energy vibrational and repulsive chemical (olfactory) barriers.

The point to be made here is that when trying to solve the containment problem in fish farming in a different way one needs to apply other engineering disciplines. The present contribution is an attempt to build a new bridge between the field of mariculture and a branch of engineering disciplines which deals with artificially controlled motion, namely the field of cybernetics with application to remotely operated underwater vehicles (ROV) and robotics.

\section{Definition of terms}

The following definitions will be applied in this contribution:

- Offshore mariculture (aquaculture) is an activity in which aquatic organisms are raised for commercial purposes under some degree of control. The term offshore

Received 15 October 1991.

Division of Engineering Cybernetics, The Norwegian Institute of Technology, University of Trondheim, Trondheim, Norway.

Paper presented at National Science Foundation Workshop on Engineering Research Needs for Off-Shore Mariculture Systems, Honolulu, Hawaii, September 26-28, 1991. 


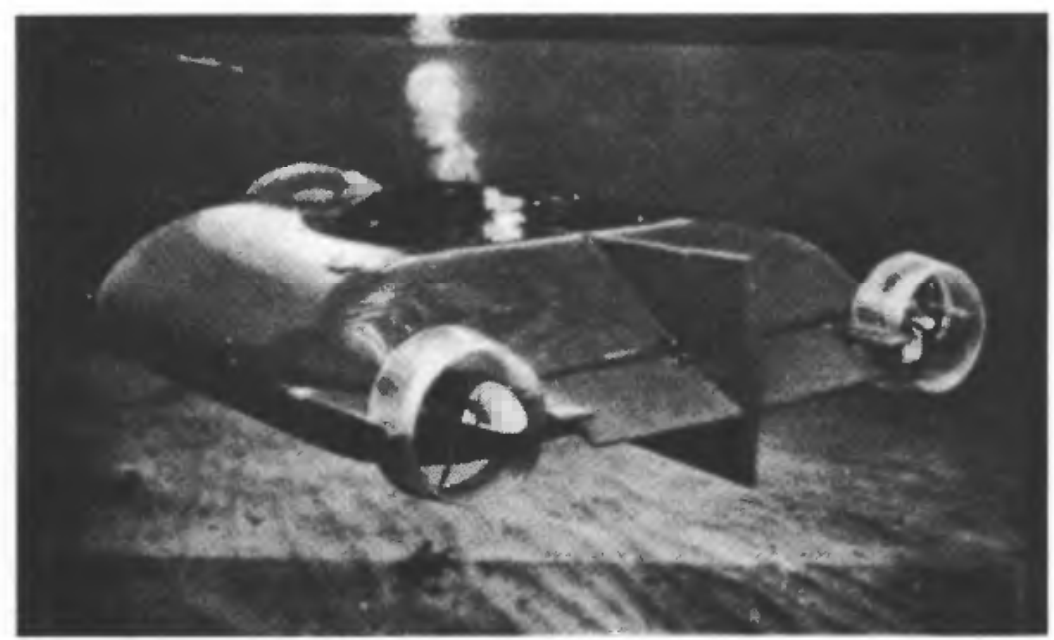

Figure 1. The SIMRAD Subsea free-swimming ROV.

relates to activities in coastal, near-coastal and mid-ocean areas in contrast to onshore activities encompassing totally artificial ponds and tanks. Where the organism feeds on natural prey the term ocean ranching is frequently used. In most cases of mariculture juveniles bred in hatcheries are planted in the area.

- A remotely operated underwater vehicle (ROV) is a mechanical device driven by a number of thrusters capable of performing motion in many degrees of freedom (often 6) under the control of an operator. An ROV may be tethered (has cable for energy and/or information) or untethered (Fig. 1).

- An ROV may carry one or more manipulator arms (robotic arms) capable of performing independent motion of its end effector in many degrees of freedom (often 6) (Fig. 2).

- An autonomous underwater vehicle (AUV) is a free swimming underwater vehicle capable of performing motion and certain operations without the aid of an operator and is only based upon local 'intelligence'.

- A semi-autonomous underwater vehicle (SAUV) is a device which combines some of the features of an AUV and an ROV.

- Robotics deals with the science and technology of controlled motion of mechanical devices, particularly manipulator arms capable of controlling an end effector (hand) to perform a useful operation such as handling, machining, assembly etc. The robotic manipulator may be controlled by an operator or have some degree of autonomy based on local sensors and 'intelligent' control programs.

\section{Possible trends in mariculture methodology}

The methods currently employed in mariculture are the results of many years of development. The trends of development follow slightly different patterns in various countries and cultures and it is worth considering the governing forces behind these development tendencies. In our discussion we shall regard mariculture in a broad sense ranging from intensive fish farming etc. in relatively small enclosures with a supply of man-made diets to the ocean ranching of different species, based on the stocking of fry 


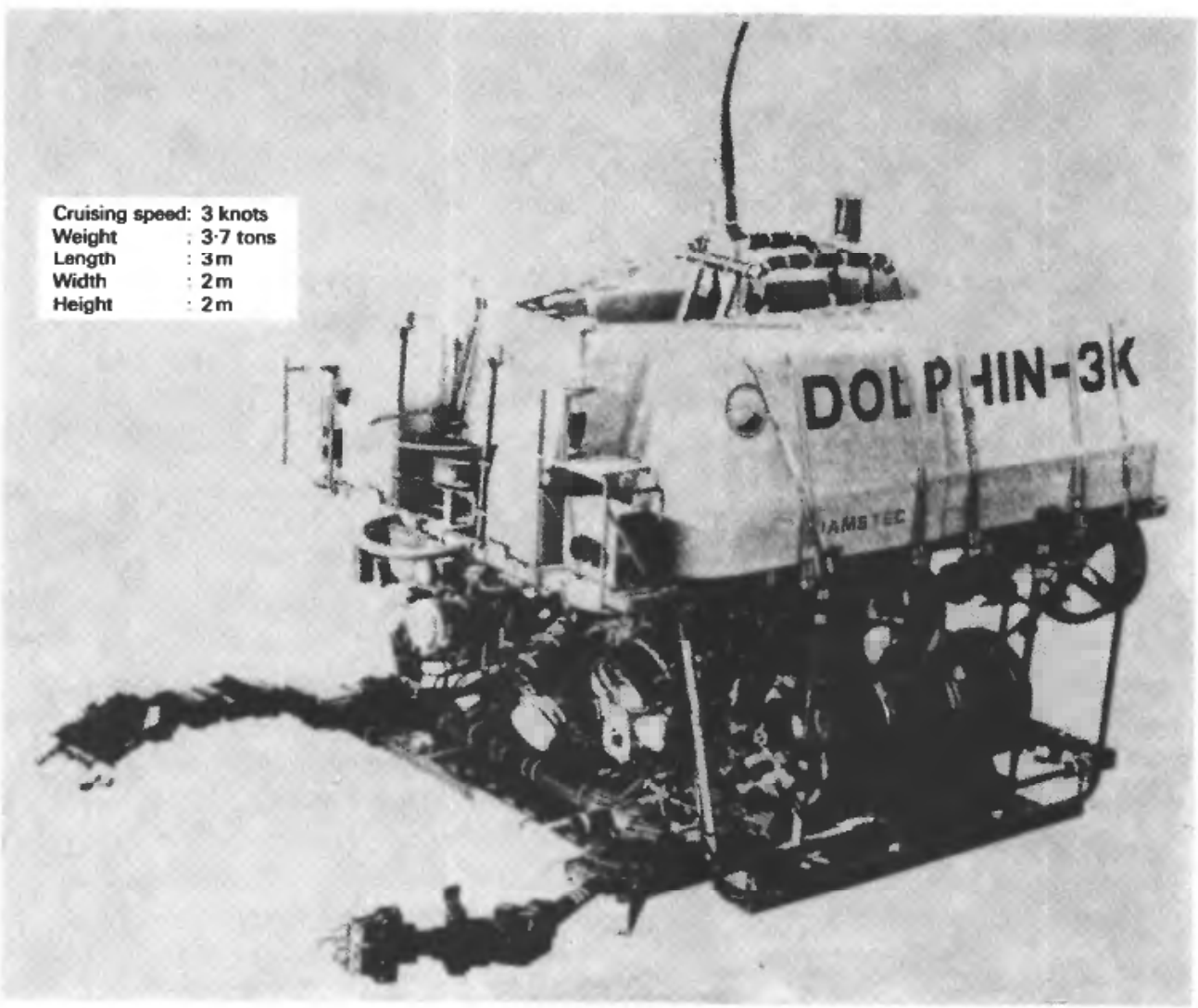

Figure 2. The Dolphin-3K ROV with two manipulator arms.

and juveniles which have been bred in hatcheries but are fed on natural prey and only have natural restrictions on migration.

The choice of methodology is a result of economic considerations such as the return of investment, cost of equipment, feed and labour and perhaps above all the influence of government laws and regulations which are very different from nation to nation. The laws of nature (physics and biology) govern what is possible and not possible but few mariculture systems are operating with such extreme limitations. The laws of nature mainly influence what has been achieved so far. More research and development will extend the achievable towards a natural limit which may be far away.

An example of the very strong governing forces imposed by local (national) regulations, is the volume limitation imposed by Norwegian authorities on salmon farming in the 1970s. The background was that Norwegian authorities decided that it was necessary to get control of the expansion of the salmon farming industry in order to realize political and economic objectives. Based upon advice from a marine research institute, it was decided to impose limitations on the volume of free floating ocean cages in order to restrict the production of each fish farmer. It was thought that the fish farmers would limit themselves to having around 15 kilos of salmon per $\mathrm{m}^{3}$ of water as recommended by the research institute. Fish farmers should apply for permission to install fish farms according to this principle and the authorities would thus have control over developments. In the beginning $2000 \mathrm{~m}^{3}$ was set as a convenient limit for one permit. This should give around 30 tonnes of salmon per permit. What the authorities did not consider was that most fish-farmers would try to put more salmon in each cage 
than the recommended $15 \mathrm{~kg} / \mathrm{m}^{3}$ and it is known that some farmers even reached a density of around $70 \mathrm{~kg} / \mathrm{m}^{3}$. This led to a number of new problems that had not been considered previously. The most pertinent are stress on the fish, mechanical wear due to close contact with the net, high pollution rate and the high probability of spreading disease. When a variety of new diseases appeared in fish farms, the fish farmers had to apply medical treatment, thereby introducing other problems that had not previously been considered. The background for introducing the volumetric limitation on the cages was to limit the biomass production, this could have been done in many other ways that did not give undesirable side effects.

One severe effect of detailed government regulations such as described above, is that technological developments can be forced in directions which may be difficult to escape from at a later stage.

In order to avoid some of the disadvantages of the Norwegian floating cage technology the limits on volume were first increased to $6000 \mathrm{~m}^{3}$ and then to $8000 \mathrm{~m}^{3}$ and $12,000 \mathrm{~m}^{3}$. Engineering research and development has been focusing on how to make bigger and better net cages. The basic idea of the net cage is to keep the fish in captivity. However the net cage is not necessarily the best way to keep fish enclosed when the volume is increased to the extent it should be in order to avoid some of the serious negative effects. In fact, Norway has no limitation on water volume and a technology utilizing the natural enclosures such as bays, sounds and fjords would be very feasible for fish farming if some kind of nonmechanical barrier could be developed for restraining the fish from migrating out of the area. Such barriers have been studied in Norway for a long time but never put into practical use because of the above mentioned volume limitations.

Another factor that supports the status quo is the interesting conserving mechanism that comes into effect through the fish farmers and their trade organizations who actually do not appear to be interested in technological progress of any magnitude because of their own investment in the previously accepted technology. These groups appear to be most interested in having a well-regulated technology so that they know what their competitors can do since they are forced to use the same methods. What does not seem to be understood is that fish farmers in other countries with other regulations may utilize entirely different technologies and thereby introduce new and more serious competition.

A conclusion to be drawn from the above discussion is that government agencies should be very careful about regulating mariculture technologies in detail because they thereby introduce an unintentional and unqualified influence upon scientific, technological and economic development.

The following issues are decisive for success in mariculture:

Yield in animal production per unit of investment.

Quality of product.

Amount of effort and resources necessary for success.

In resolving these issues one of the dominant problems becomes that of controlling the motion of the animal in question (migration).

The control of animal motion can be achieved in a number of different ways depending on the distances in question. The main methods are (Balchen 1979):

Barriers; passive and active,

Conditioning, training, learning,

Utilizing natural 'signals' in the ocean and natural preferences. 
The most obvious barrier is the mechanical net which has probably been used for thousands of years. The main disadvantage of the net is its tendency to collect debris and become a substrate for the growth of algae and mussels. Thereby it will become a hydrodynamic restriction so that large forces will develop due to currents and waves. Using nets as barriers to close off bays, sounds and fjords has also proven to be difficult for the same reasons. Thus nets are most useful in making small volume enclosures.

A number of nonmechanical barriers have been developed which do not have the disadvantages of nets. Such a barrier is the electric barrier consisting of electrodes that discharge high current pulses at the same instant as a light pulse is emitted from a source on the electrodes as illustrated in Fig. 3. The action of this barrier is based on punishment and visual learning so that the fish believes that the pain caused by the electric pulse comes from the light source from which it has directional information. Electric barriers can be used over large distances, but it must be observed that the energy consumption in salt water is not insignificant. Thus it may be advantageous to combine the electric barrier with some other type of barrier.

Many species of fish react with repulsion to certain high energy, low frequency vibrations which can be generated by mechanical, hydraulic or electrical vibrators.

Air bubble curtains are known to act as barriers for certain species like herring, saith and others. Salmon on the other hand, do not react to an air-bubble curtain. It has been proposed mixing a chemical agent in small quantities into the air to which the fish will react repulsively. Such chemical agents can be extracted for example from natural predators of the fish or they can be natural substances such as the hydrogen sulphide gas $\left(\mathrm{H}_{2} \mathrm{~S}\right)$.

An 'intelligent' barrier is one that is only activated when there is a need, that is when a fish is approaching the barrier on its way out of the area. The barrier consists of a

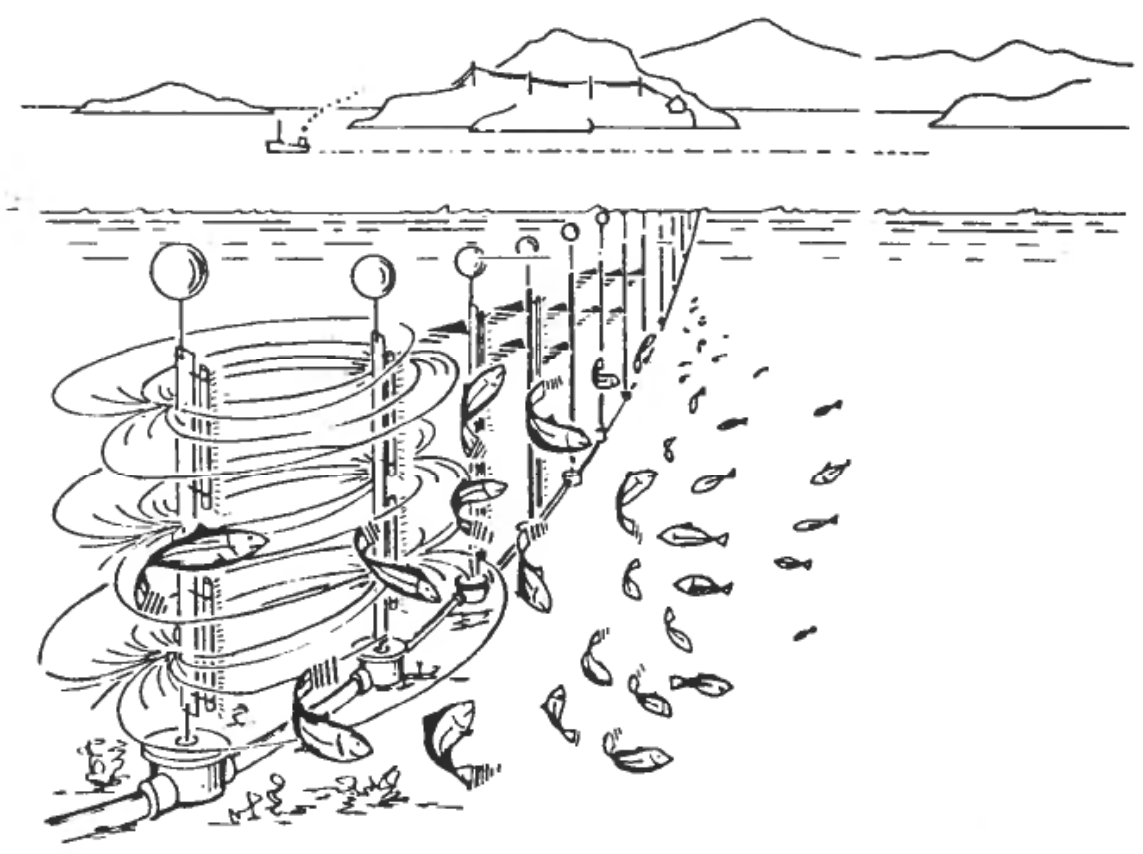

Figure 3. An electrical barrier based on conditioning with light pulses as signals and electric pulses as 'punishment'. 
detector (ultrasonic) which senses the proximity of the fish and activates the barrier which may be either electrical, vibro-acoustical, or an air-operated net.

Conditioning has proven to be very powerful in controlling animal behaviour, particularly fish behaviour. In conditioning, two 'signals' are involved, one which transmits rapid long-distance signals which can easily be detected by the animal and a second which is 'meaningful' (attractive = food, painful=electric shock). The electric barrier explained above works on the principle of conditioning (Balchen 1981).

Experiments have been performed with conditioning fish in fairly large areas (approx. $1 \mathrm{~km}^{2}$ ) by using sound and food. If sound signals (approx. $200 \mathrm{~Hz}$ ) are transmitted at precise intervals (say 10 minutes) followed by a portion of the favourite feed of the fish, the fish will be systematically attracted and it takes a very short time before it learns the relationship between the sound and the feed. Conditioning appears to be an important part of the learning and experience of young fish and plays a role in its natural migration at a later stage. In conditioning acoustic, visual and olfactory stimuli are possible choices of 'signals' (Balchen 1979).

\section{Fields of application of ROVs and robotics in mariculture}

Maybe the most obvious application of an ROV in mariculture is that of inspection of a mariculture facility. Modern instrumentation technology including imaging by means of cameras and ultrasonic devices facilitates the extension of the human senses from the surface to the underwater world. Inspection includes the visual imaging as well as the collection of data about temperature, water quality, water motion, counting the number of individual organisms, estimating biomass etc. Since the ROV can be steered to any position in the mariculture facility, samples may be taken of the water and even individual organisms that can be brought to the surface for inspection and analysis. The obvious alternative to this technology is the use of human divers with their limitations with regard to depth, temperature, safety and even the cost of operation (Balchen 1986, Klepaker et al. 1986).

Another important application of ROVs and robotics is in the handling of objects and the operation of devices. Such tasks can be divided in two categories namely routine and extraordinary operations. Among routine operations are tasks like operating valves, moving and connecting objects and installing equipment and performing simple repair tasks such as mending nets in cases of malfunction and emergency. The primary reason for the possible application of ROVs and robotics for these tasks is that they can be performed at greater depths than is possible with human divers and in general with less hazard to human lives.

An example of an application of a remotely operated underwater vehicle is in the inspection, maintenance and operation of a fully submerged cage system as indicated in Fig. 4 (Osborne et al. 1990). Fully submerged cages have been suggested for some time and have been partly tested in Norway. The motivation for fully submerged cages is that they are less subject to forces imposed by weather and the net material will experience less growth of algae if the system is located 7-10 $\mathrm{m}$ below the water surface. For physiological reasons species like salmon need to have access to air and therefore the upper part of the conical net has an airfilled top.

A system as shown in Fig. 4 will need attendance which can be performed by divers, but this is costly and hazardous, and here a ROV controlled from a surface platform may be relevant. The ROV can be used for moving the net cage to a different position on the bottom, mending net damage, inspecting the fish population, estimating its 
condition, biomass and number, interacting with the fish population for the purpose of medication etc., and even for harvesting parts of the population.

Obviously the technical development of such an ROV system will involve certain unsolved problems, but it is a matter of analysis to find the technical, biological and economic feasibility of such systems. So far this does not appear to have been done.

Another, more long-term application of remotely operated underwater vehicles in mariculture is inspired from experiments that have been conducted in Norway for a long time on the use of conditioning in the control of fish behaviour. It has been clearly demonstrated that a number of the species of fish that are important for mariculture can be made to display schooling behaviour. In a large natural area (say $5-10 \mathrm{~km}^{2}$ ) surrounded by natural barriers like islands, it is feasible to make large quantities of fish perform programmed migration between fixed feeding stations as indicated in Fig. 5 . The motion of the fish is induced by a conditioned response to an acoustic signal coming from the feeding stations in a travelling type of wave pattern. In such a system a free swimming ROV may play the role of a 'shepherd' whose function it is to control straying individuals back into the flock by the application of mild repulsive or preferably attractive signals. Echo-sounding and sonar technology, which has become inexpensive in recent years, is directly applicable using the ROV for the detection of the location of the fish.

A somewhat related application for an ROV would be as a 'leader' of a school of fish in an open area applying conditioning with sound and feed. The purpose of such a system could be to utilize natural prey in a mariculture context. Possibly a number of ROVs could interact in such a system by locating themselves in different positions around the fish population, thereby achieving better control of the motion of the fish.

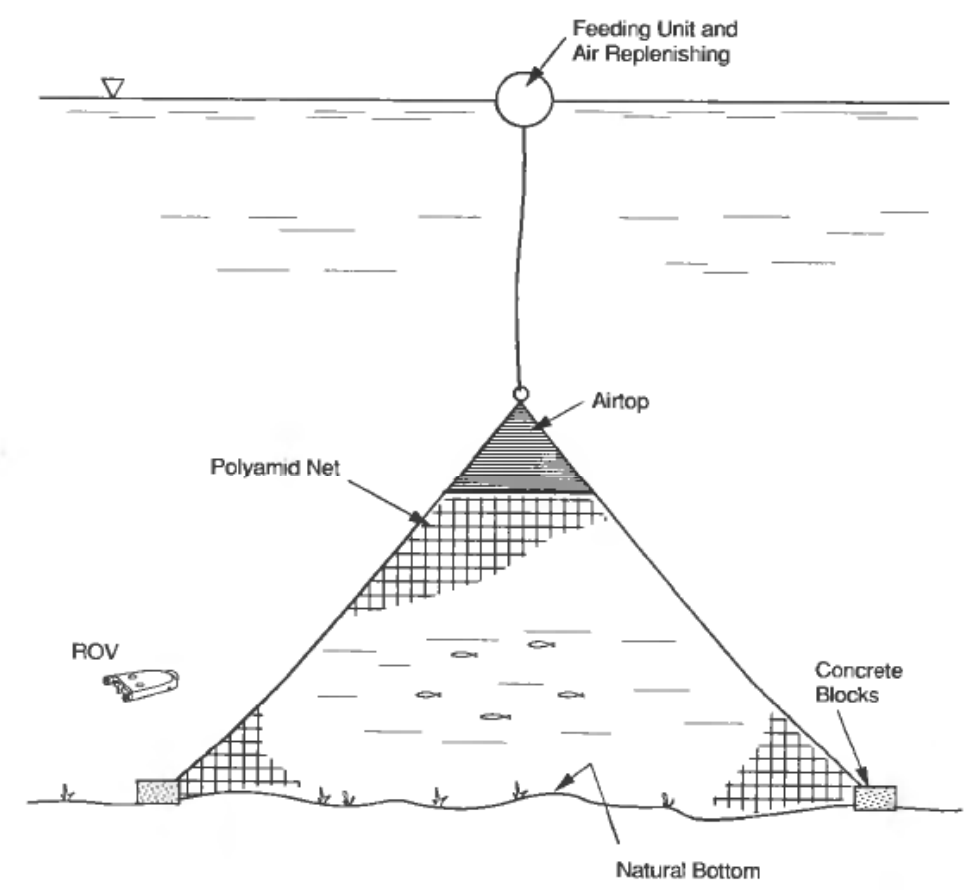

Figure 4. A fully submerged cage system proposed for fish farming. 


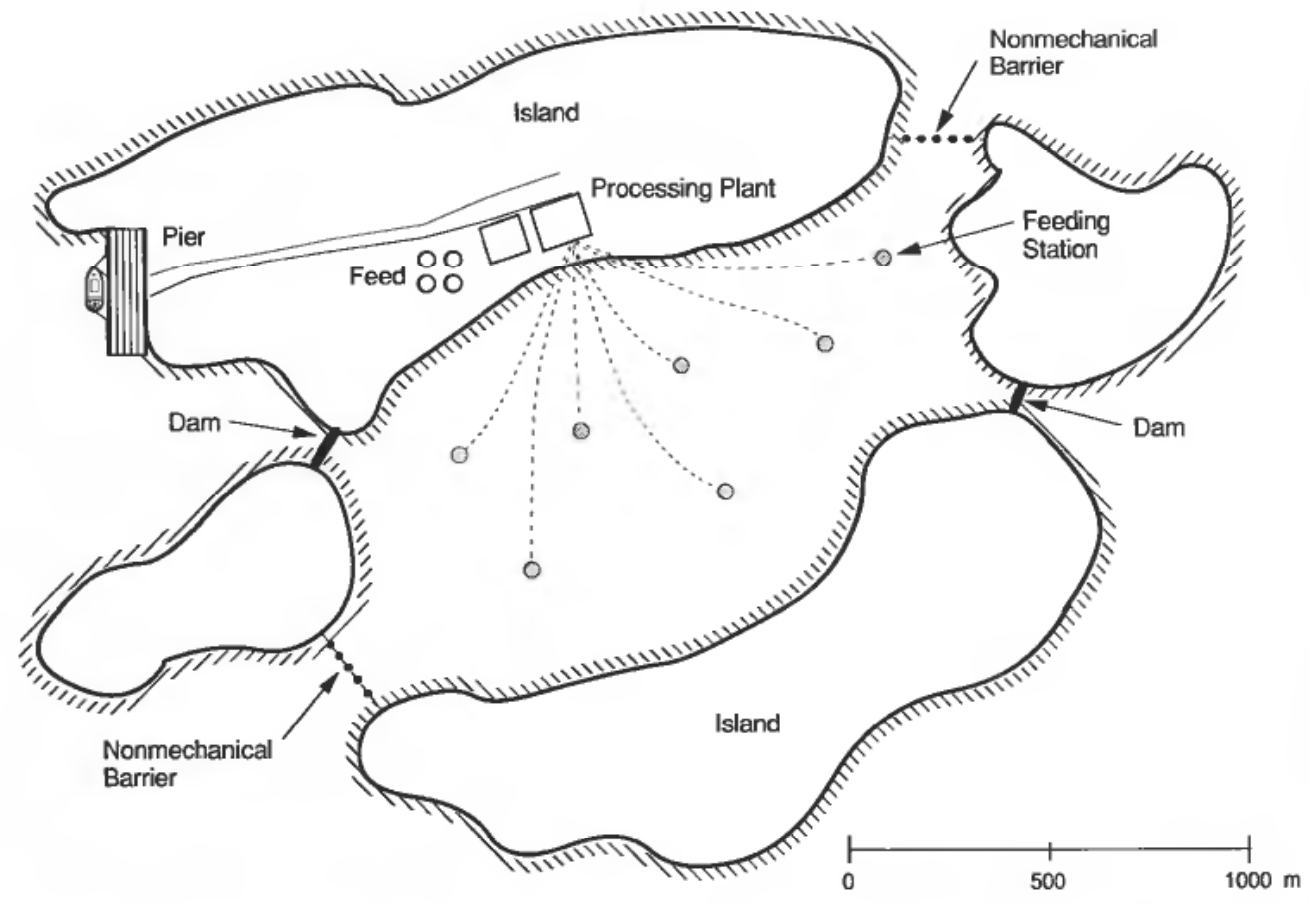

Figure 5. Fish farming in natural closed-off waters employing behaviour control by conditioning.

One field of the application of remotely operated underwater vehicles is in the future large-scale farming of lobsters which is under development in several places, including Norway. Large numbers of juvenile lobsters are to be planted in natural but highly selected areas where conditions are the best for the lobsters to thrive and survive. Finding such areas, recording their location in detail and later on releasing the juveniles in these locations is an obvious task for a remotely operated underwater device. Such a device can take many forms and the development of a practical system is now underway in Norway. The same system with automatic dynamic positioning of the surface vessel based on the most modern navigation technology and the remotely operated underwater device is regarded as a necessary means for making tomorrow's lobster ranching economically attractive.

The application of robotic manipulators for the handling of objects in mariculture systems has been mentioned above. Another field of great interest which is related to this is the use of robotic devices combined with imaging systems for a variety of sorting purposes in hatcheries and juvenile production. An example of this is the automatic sorting of fourth stage lobster larvae in large scale lobster juvenile production. The problem is that quite a high percentage of the tiny lobsters after the fourth moulting $(2-3 \mathrm{~cm})$ have some defect and should be sorted out. One such defect is the lack of a claw. In large-scale production this must be done automatically by the application of modern imaging equipment together with some kind of mechanical sorting. This is within reach. Similar problems exist in sorting out dead eggs in fish hatcheries (Balchen 1989).

Even though the processing of the mariculture product, such as fish, does not necessarily belong to mariculture itself, some very interesting new application of robotics have been presented in recent years. One such development is a robot-like 
machine for removing the intestines and cleaning a fish without opening the stomach. The success of automating this particular process is expected to be a major contribution to securing the quality of the fish product.

\section{Some aspects of ROV technology}

Remotely operated underwater vehicles can take many forms and sizes and have numerous kinds of control systems. One frequently distinguishes between different technical categories by the degree of energy autonomy and the degree of information autonomy. A vehicle which is totally supplied by energy from a source on board the vehicle is said to be 100 per cent energy autonomous. On the other hand, if the energy is supplied through a cable, it has 0 per cent energy autonomy.

A heavy cable to supply energy is a major disadvantage because it hinders free motion and consumes a lot of energy because of the drag that water currents impose on the cable. Reliable, inexpensive and compact energy sources offering both high power and high energy are not easily available at the moment. Therefore intensive research is underway in many countries to develop better solutions. Rechargeable batteries such as the standard lead-acid battery is the most commonly used in inexpensive vehicles. Much more compact, powerful battery units are presently available at a much higher price, but the price if falling rapidly and may reach an acceptable level fairly soon. Larger energy units (of $50 \mathrm{~kW}$ and over) are available based on different kinds of rotating machinery with liquid fuel as the energy source. Major progress is expected in this sector in the near future.

Information autonomy expresses the degree to which the vehicle is capable of performing its own control and decision making. When such a vehicle is 100 per cent autonomous no human skill or intelligence is involved and the vehicle operates entirely on its own. At the other end of the scale 0 per cent information autonomous means that a human operator is in charge of all 'intelligent' functions. In most cases it is of interest to have a human operator perform the most important supervisory control functions and decision making whereas the vehicle has some local 'intelligence' in the form of control systems based on the measurement of position and velocity in 6 degrees of freedom and even the processing of images generated by local cameras etc. If the vehicle has less than 100 per cent information autonomy, it needs a communication system to communicate with the human operator on the surface. This communication may utilize a cable (electrical or fibre optic) which represents no problem if a power cable is available anyway. If the vehicle is 100 per cent energy autonomous, the communication may either go through a thin elecrical cable, a fibre optic cable or via an ultrasound transmission system. The latter has the advantage of being totally free of physical contact and thereby giving the vehicle total freedom to move. Since an ultrasonic communication system will have a very low communication capacity (low signal bandwidth) there is a dramatic need for image compression. This is a technique for extracting the real information from an image and transmitting only this part, leaving behind perhaps 99 per cent of what is usually contained in an image. This problem has attracted many researchers during the last decades and some remarkable results have been obtained. Combining the excellent capability of the human being to recognize patterns with the capabilities of modern imaging technology, powerful data compression algorithms, robust, but sophisticated ultrasonic data transmission it is now possible to implement control of an underwater vehicle which performs as if the 


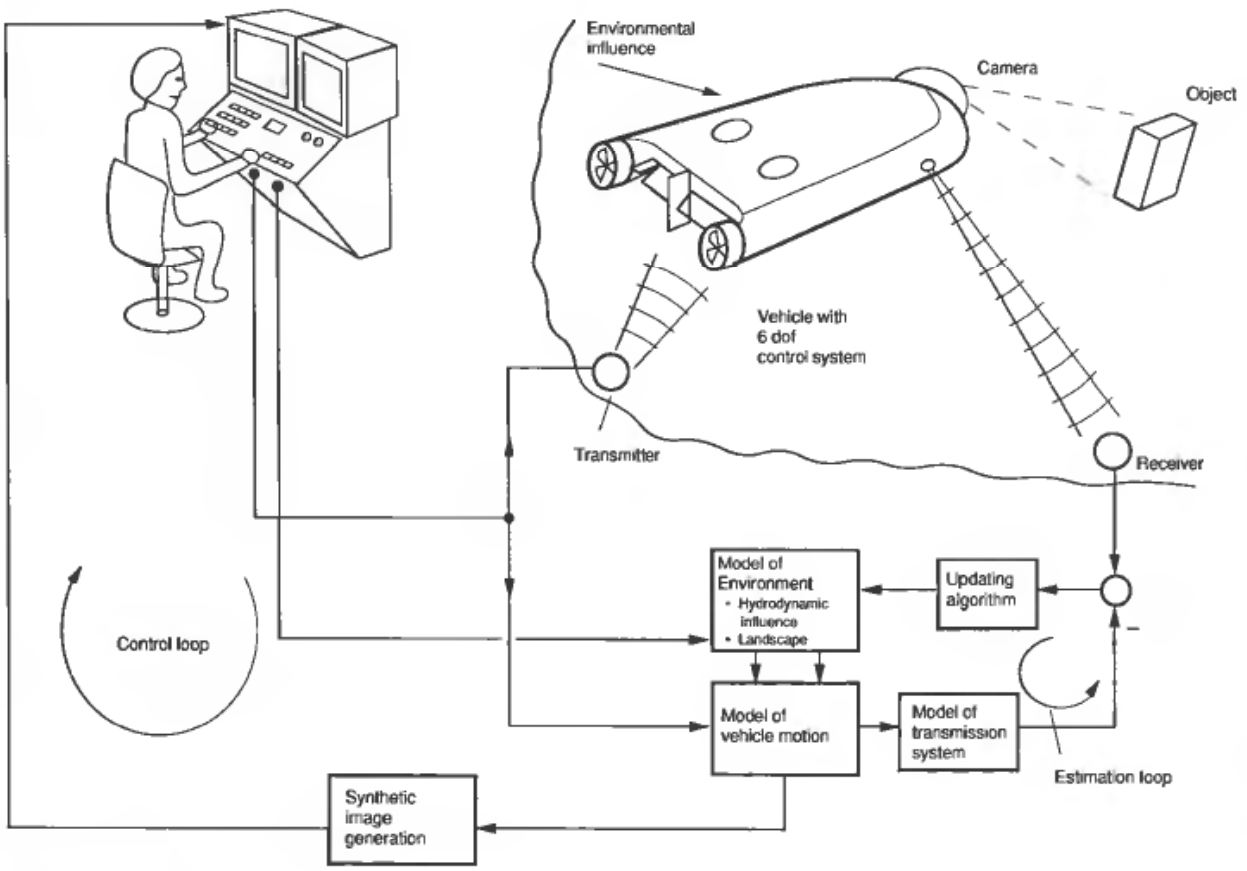

Figure 6. The MOBATEL system for model-based teleoperation of an underwater vehicle over a narrow band communication link.

operator were sitting in the vehicle with visual contact. A very powerful method referred to as model-based teleoperation utilizes mathematical models of vehicle motion and geometric models of underwater landscapes which are updated via the imaging devices on board the vehicle and thereby making it possible for the operator to control the motion based on synthetically generated images. Research in this field is presently underway in many countries including the MOBATEL-project in Norway. A block diagram depicting the functional elements involved in the MOBATEL-system is shown in Fig. 6.

\section{Conclusions}

The dramatic progress which has been made in technological systems related to remotely operated underwater vehicles and robotics offers many possibilities for fruitful applications in the mariculture context. In order to fulfil this promise there is a need for elaborate, maybe painstaking exercises to remove difficulties not thought of during the development of the fundamental concepts. Above all there is a need to loosen the ties to the presently accepted technologies and return to the definition of the original problem. Research based on marginal improvements usually gives one particular development line which may not turn out to give the best results. The availability of the new technologies may make entirely new mariculture concepts possible. These include ocean ranging with behaviour control and the utilization of large naturally enclosed water masses without artificial mechanical barriers. 


\section{REFERENCES}

Balchen, J. G. (1979). Modeling, Prediction and Control of Fish Behavior. In Control and Dynamic Systems, ed. C. T. Leondes (Academic Press Inc.).

Balchen, J. G. (1981). Recent Progress in the Control of Fish Behaviour. Proc. IF AC 8th World Congress, Kyoto, Japan (Pergamon Press, Oxford).

BALCHEN, J. G. (1986). Bridging the Gap Between Aquaculture and the Information Sciences. Proc. IF AC Symp. on Automation and Data Processing in Aquaculture (IFAC Proc. No. 9, 1987) (Pergamon Press, Oxford).

BALCHEN, J. G. (1989). Instrumentation, Information and Control Systems in Aquaculture. Proc. Spec. Sess. of World Aquaculture Society 1989 Anmual Meeting (Oceanic Institute, P.O. Box 25280, Honolulu, Hawaii).

KlePAKer, R. A., VestgÅrd, K., Hallseth, J. O., and BAlChen, J. G. (1986). The application of a Free-Swimming ROV in Aquaculture. Proc. IFAC Symp. on Automation and Data Processing in Aquaculture 1986 (IFAC Proc. No. 9, 1987) (Pergamon Press).

Osborne, H. D., Eadie, H. S., FunNel, C., Kuo, C., and Linfoot, B. T. (1990). Engineering for Offshore Fishfarming. Proc. of Conf. organized by the Inst. of Civil Eng., Glasgow (Thomas Telford, London). 